\title{
Pre-pregnancy dietary patterns and risk of gestational diabetes mellitus: results from an Australian population-based prospective cohort study
}

\author{
Danielle A. J. M. Schoenaker ${ }^{1}$ - Sabita S. Soedamah-Muthu ${ }^{2}$. \\ Leonie K. Callaway ${ }^{3,4} \cdot$ Gita D. Mishra $^{1}$
}

Received: 18 May 2015 / Accepted: 10 August 2015 / Published online: 10 September 2015

(C) Springer-Verlag Berlin Heidelberg 2015

\begin{abstract}
Aims/hypothesis We examined the associations between prepregnancy dietary patterns and the incidence of gestational diabetes mellitus (GDM) in a population-based cohort study of women of reproductive age.

Methods The Australian Longitudinal Study on Women's Health included 3,853 women without pre-existing diabetes who were followed-up between 2003 and 2012. Prepregnancy dietary patterns were derived using factor analysis based on 101 food items from a validated food frequency questionnaire. GDM was self-reported and validated in a subsample. Multivariable regression models with generalised estimating equations were used to estimate RR and 95\% CI. Results During 9 years follow-up, 292 GDM cases (4.4\%) were documented in 6,626 pregnancies. No associations were found for the 'Fruit and low-fat dairy' and 'Cooked vegetables' patterns. The 'Meats, snacks and sweets' pattern was associated with higher GDM risk after adjustment for socioeconomic, reproductive and lifestyle factors (RR $[95 \% \mathrm{CI}]$ per
\end{abstract}

Electronic supplementary material The online version of this article (doi:10.1007/s00125-015-3742-1) contains peer-reviewed but unedited supplementary material, which is available to authorised users.

Danielle A. J. M. Schoenaker

d.schoenaker@uq.edu.au

School of Public Health, University of Queensland, Herston Road, Herston, QLD 4006, Australia

2 Division of Human Nutrition, Wageningen University, Wageningen, The Netherlands

3 School of Medicine, University of Queensland, Brisbane QLD, Australia

4 Departments of Obstetric and Internal Medicine, Royal Brisbane and Women's Hospital, Brisbane, QLD, Australia
SD increase in score: $1.38[1.02,1.86])$. Further adjustment for BMI attenuated the results $(1.35[0.98,1.81])$. In stratified analysis, the 'Meats, snacks and sweets' pattern was associated with significantly higher GDM risk in parous and obese women, and in women with lower educational qualifications. The 'Mediterranean-style' pattern was associated with lower GDM risk in the fully adjusted model $(0.85[0.76,0.98])$. Conclusions/interpretation These findings support general dietary recommendations for women of reproductive age to consume a diet rich in vegetables, whole grains, nuts and fish, and low in red and processed meats and snacks. Further prospective studies are needed to confirm these findings.

Keywords Dietary patterns - Gestational diabetes mellitus . Nutrition · Pregnancy

$\begin{array}{ll}\text { Abbreviations } \\ \text { ALSWH } & \begin{array}{l}\text { Australian Longitudinal Study on Women's } \\ \text { Health }\end{array} \\ \text { FFQ } & \text { Food frequency questionnaire } \\ \text { GDM } & \text { Gestational diabetes mellitus } \\ \text { GEE } & \text { Generalised estimating equation } \\ \text { MET } & \text { Total metabolic equivalent }\end{array}$

\section{Introduction}

Gestational diabetes mellitus (GDM) is one of the most common pregnancy complications, affecting approximately $7 \%$ of pregnancies $[1,2]$. The prevalence of GDM has increased over the last 20 years and is expected to rise further as a result of the higher number of women entering pregnancy as overweight or obese [3]. Women with GDM are at increased risk of complications during pregnancy and delivery [4]. 
Following pregnancy, more than 50\% of women with GDM in pregnancy develop type 2 diabetes within 5 to 10 years [5]. Moreover, the risks of childhood obesity and early onset of type 2 diabetes are elevated in offspring of women with GDM [6], and female offspring are more likely to develop GDM themselves [7]. Collectively, this evidence highlights that identification of modifiable risk factors for GDM may have significant clinical and public health implications and may contribute to reducing inter-generational adverse health outcomes in mothers and their children.

Findings from trials aiming at reducing the risk of GDM through dietary interventions during early pregnancy are suggestive of a beneficial effect [8,9]. Epidemiological evidence of the association between dietary intake and GDM is sparse and predominantly focused on intake of single nutrients and foods [10]. Our recent systematic review of observational studies indicated an increased risk of GDM associated with high consumption of total fat, cholesterol and haem iron [10]. Moreover, high consumption of red meat, processed meat and eggs were identified as risk factors. Limited attention has been given to the role of overall dietary patterns in the development of GDM. Furthermore, in addition to the importance of a healthy diet during pregnancy, nutrition and lifestyle prior to pregnancy have been suggested to be crucial for optimal onset and development of pregnancy $[11,12]$. So far, only studies using data from the Nurses' Health Study II have examined dietary patterns before pregnancy in relation to GDM risk [13-15]. These findings need confirmation in other study populations to draw conclusions on the role of pre-pregnancy diet in the development of GDM.

The objective of this study was to examine the associations between pre-pregnancy dietary patterns and the risk of GDM in a population-based study of women of reproductive age.

\section{Methods}

\section{Study design and participants}

Study participants were from the Australian Longitudinal Study on Women's Health (ALSWH). ALSWH is an ongoing population-based prospective cohort study of factors affecting health and wellbeing of Australian women. Women were randomly selected from the national Medicare health insurance database, which covers all Australian citizens and permanent residents. Women living in rural and remote areas were intentionally oversampled. Full details on study design, recruitment methods and responses have been published previously [16, 17]. Informed consent was obtained from all participants at each survey and the study was approved by the Human Research Ethics Committees at the University of Newcastle and the University of Queensland, Australia.
This study used data from the young cohort of women born between 1973 and 1978. A total of 14,427 women aged 18-23 years responded to the first survey in 1996. This sample was found to be broadly representative of the general Australian population of women of similar age [16]. Selfadministered questionnaires were sent to participants every 3-4 years. Dietary intake was first collected at Survey 3 in 2003 when women were aged $25-30$ years $(n=9,081)$ and this survey was therefore used as baseline in the present study. Data were excluded of women who were pregnant at the time of Survey 3, who did not report a live birth at consecutive surveys in 2006 (Survey 4, age 28-33 years), 2009 (Survey 5, age 31-36 years) or 2012 (Survey 6, age 34-39 years), had missing data on diet or GDM, reported implausible energy intake $(<2,093 \mathrm{~kJ}$ or $>14,654 \mathrm{~kJ}$ [18]), had a history of type 1 or type 2 diabetes or GDM, or had missing covariate data (Fig. 1). Our analyses included 3,853 women.

\section{Dietary assessment}

Diet was assessed using the Dietary Questionnaire for Epidemiological Studies version 2. The development of this 101-item food frequency questionnaire (FFQ) has been described elsewhere [19]. The FFQ was validated against 7 day food diaries for 63 reproductive-aged women who participated in an iron deficiency study [20]. Energy-adjusted correlation coefficients for a wide range of macro- and micronutrients were moderate to strong (ranging from 0.28 for vitamin A to 0.78 for carbohydrates). Mean intakes from the food dairies and FFQs were within 20\% for 21 of the 27 nutrients that were estimated. These results represent performance comparable to other widely used FFQs [21, 22]. Participants were asked to report their usual frequency of consumption of 74 food items and six alcoholic beverage items over the previous 12 months using a 10-point scale ranging from 'Never' to 'Three or more times per day'. Portion size photographs were used to adjust the serving sizes for vegetables, meat and casseroles. The FFQ additionally included 21 items on the number of servings of milk, bread, sugar and eggs, and the type of milk, bread, fat spread and cheese consumed. Consumption of food items (in grams per day) and nutrient intakes were computed from the national government food composition database of Australian foods, the NUTTAB95 [23].

\section{Assessment of GDM}

GDM was ascertained from self-reported physician diagnosis at each survey and for each live birth using the following question: 'Were you diagnosed or treated for gestational diabetes?'. Women with GDM up to Survey 3 were excluded for the present study. Incidence of GDM was defined as new cases between Survey 3 (2003) and Survey 6 (2012). During 
Fig. 1 Flow diagram of the sample for analysis of the association between prepregnancy dietary patterns and the risk of GDM

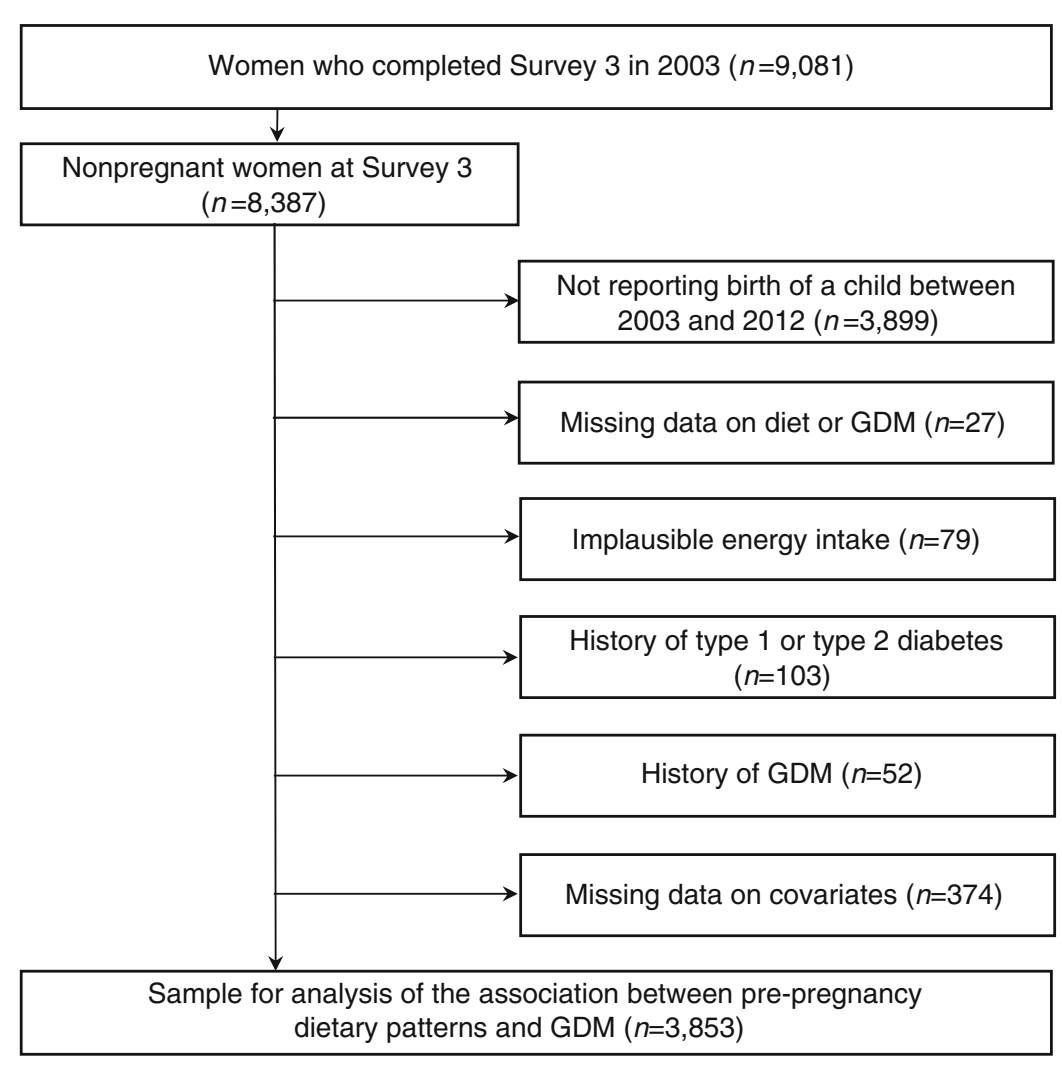

the study period, diagnostic criteria for GDM in Australia included a $1 \mathrm{~h}$ venous plasma glucose level $\geq 6.55 \%$ $(7.8 \mathrm{mmol} / \mathrm{l})$ after a $50 \mathrm{~g}$ glucose load, or a $1 \mathrm{~h}$ venous plasma glucose level $\geq 6.65 \%$ ( $8.0 \mathrm{mmol} / \mathrm{l})$ after a $75 \mathrm{~g}$ glucose load. Diagnosis was confirmed with a $75 \mathrm{~g}$ OGTT (fasting) with a venous plasma glucose level at $0 \mathrm{~h}$ of $\geq 5.1 \%(5.6 \mathrm{mmol} / \mathrm{l})$ and/or at $2 \mathrm{~h}$ of $\geq 6.65 \%$ ( $8.0 \mathrm{mmol} / \mathrm{l})$ [24]. A reliability study among a subgroup of women from New South Wales $(n=1,914)$ demonstrated high agreement of $91 \%$ between self-reported GDM diagnosis in our study and administrative data records [25].

\section{Assessment of covariates}

Self-reported information on highest qualification completed, parity, hypertensive disorders of pregnancy, polycystic ovary syndrome, inter-pregnancy interval, smoking, physical activity and BMI was collected at baseline (Survey 3 ) and repeatedly during follow-up surveys when pregnancy outcomes were indicated (Survey 4 to Survey 6). Physical activity scores were derived from validated questions on frequency and duration of walking and on moderate- and vigorous-intensity activity and categorised as sedentary/low ( $<600$ total metabolic equivalent $[\mathrm{MET}] \mathrm{min} /$ week), moderate (600 to $<1200 \mathrm{MET}$ $\mathrm{min} /$ week) or high ( $\geq 1200 \mathrm{MET} \mathrm{min} /$ week) [26]. BMI was categorised as underweight (BMI $<18.5 \mathrm{~kg} / \mathrm{m}^{2}$ ), healthy weight
(BMI 18.5 to $<25 \mathrm{~kg} / \mathrm{m}^{2}$ ), overweight (BMI 25 to $<30 \mathrm{~kg} / \mathrm{m}^{2}$ ) or obese $\left(B M I \geq 30 \mathrm{~kg} / \mathrm{m}^{2}\right)$. Only a few women were classified as underweight ( $n=162,4.3 \%$ ); therefore, they were combined and classified as healthy weight $\left(\mathrm{BMI}<25 \mathrm{~kg} / \mathrm{m}^{2}\right)$.

\section{Statistical analysis}

Pre-pregnancy dietary patterns based on 101 food items were identified using exploratory factor analysis with the principal component method and Varimax/orthogonal rotation. Food items were not pre-grouped into broader food groups to reduce the number of arbitrary decisions to be made, to allow for completely data-driven dietary patterns based on correlation between all food items, and because greater detail in dietary patterns may be important for more precise estimates of disease risk [27]. Eigenvalues $\geq 1.25$, identification of a break point in the screeplot, and interpretability were used to determine the number of dietary patterns that best represented the data [28]. Factor loadings represent the correlation between food items and a particular pattern. Factors were labelled according to those foods that contributed most to the pattern (factor loading $\geq 20$ ).

Baseline characteristics were described for women who did and did not develop GDM during follow-up, and for women in the bottom and top tertiles of dietary pattern scores, and compared using $t$ tests, ANOVA or $\chi^{2}$ tests. All descriptive 
statistics were weighted by area of residence to account for the oversampling of women from rural and remote areas.

Generalised estimating equations (GEE) analyses, which account for correlations in repeated pregnancies contributed by a single woman [29], were used to examine the prospective associations between pre-pregnancy dietary patterns (Survey 3) and incident GDM (Survey 4-6). Log-binomial models or log-Poisson models (when logbinomial models did not converge) were used to estimate $\mathrm{RR}$ and $95 \% \mathrm{CI}$. We adjusted for time varying covariates (age, highest qualification completed, lifestyle [smoking and physical activity], BMI and polycystic ovary syndrome) reported at the survey prior to the index pregnancy (Survey 3-5), and reproductive characteristics (hypertensive disorders of pregnancy, parity and inter-pregnancy interval) reported at the same survey as the index pregnancy (Survey 4-6). All models were adjusted for energy intake (kJ/day) reported at Survey 3. Country of birth, multiple gestation and previous macrosomia were not included in the models as these were not significant confounders based on the data. If a linear trend was evident from estimates across tertiles of dietary pattern scores, a $p$ value for linear trend was determined, and dietary patterns were in addition analysed as continuous scores to retain power. Dietary pattern scores were standardised and each increase of one unit therefore reflects one SD.

Additional GEE analyses were conducted to identify associations of food items that contributed mostly to significant dietary patterns (Survey 3) with incident GDM (Survey 4-6). Food items associated with GDM were then removed from the dietary pattern to examine whether the association between the pattern and GDM risk persisted. Models were adjusted for time varying covariates as described above.

Consistency of our results was tested in a range of sensitivity analyses. First, analyses were stratified by known risk factors for GDM including parity, highest qualification completed, polycystic ovary syndrome, smoking, physical activity and BMI to detect potential high-risk subgroups. Second, we compared dietary patterns identified based on data from FFQs at Survey 3 and Survey 5 to minimise the potential effect of any changes in diet over time and reduce measurement error [30]. As dietary patterns were very similar at each time point, we then calculated average long-term diet of nonpregnant women using data from both surveys, and examined the associations between average long-term pre-pregnancy dietary patterns and GDM risk. Last, we conducted a multiple imputation analysis to assess the influence of participant exclusions that resulted from missing covariate data $(n=374)$ using SAS procedures MI and MIANALYZE [31].

Statistical analyses were conducted using SAS Software Version 9.4 (SAS Institute Inc., Cary, NC, USA). A $p$ value $<0.05$ was considered statistically significant.

\section{Results}

We identified four dietary patterns using factor analysis (electronic supplementary material [ESM] Table 1). Factor loadings were rotated using the Varimax (orthogonal) method and dietary patterns are therefore independent of each other (spearman correlation coefficients $<0.05$ ). The 'Meats, snacks and sweets' pattern was characterised by high consumption of red and processed meat, cakes, sweet biscuits, fruit juice, chocolate and pizza. The 'Mediterranean-style' pattern showed high factor loadings for vegetables, legumes, nuts, tofu, rice, pasta, rye bread, red wine and fish. The 'Fruit and low-fat dairy' pattern positively correlated with fruits and lowfat dairy including yoghurt, low-fat cheese and skimmed milk. The 'Cooked vegetables' pattern was characterised by high consumption of carrots, peas, cooked potatoes, cauliflower and pumpkin. Consumption of nutrients and foods in the bottom and top tertiles for each dietary pattern is shown in ESM Table 2.

Among 3,853 women who reported at least one live birth during 9 years follow-up, we documented 292 GDM cases $(4.4 \%)$ in 6,626 pregnancies. Women who developed GDM were more likely to be nulliparous, overweight or obese, and have lower educational qualifications and a diagnosis of polycystic ovary syndrome (Table 1 ).

Women in the top tertiles of the Meats, snacks and sweets and the Cooked vegetables dietary patterns were more likely to have lower educational qualifications, be overweight or obese, and have sedentary or low physical activity (Table 2). By contrast, women in the top tertiles of the Mediterraneanstyle and the Fruit and low-fat dairy dietary patterns were more likely to have a university or higher degree, high physical activity and be never or exsmokers. High scores for the Mediterranean-style dietary pattern were also associated with a healthy weight.

\section{Pre-pregnancy dietary patterns and GDM}

No associations were found for the pre-pregnancy Fruit and low-fat dairy and the Cooked vegetables dietary patterns and GDM risk (Table 3). The Meats, snacks and sweets and the Mediterranean-style dietary patterns were associated with risk of GDM and described below.

Meats, snacks and sweets dietary pattern Each SD increase in score of the Meats, snacks and sweets pattern was associated with a $41 \%$ higher GDM risk $(95 \%$ CI $1.03,1.91)$ after adjustment for socioeconomic and reproductive factors (Table 3). This association was slightly attenuated after adjustment for lifestyle factors (RR 1.38, 95\% CI 1.02, 1.86), and no longer statistically significant after additional adjustment for BMI (RR 1.35, 95\% CI 0.98, 1.81). 
Table 1 Baseline characteristics of nonpregnant women according to prospective development of $\operatorname{GDM}(n=3,853)$

\begin{tabular}{|c|c|c|c|}
\hline Pre-pregnancy characteristic & $\begin{array}{l}\text { GDM incidence } \\
n=292\end{array}$ & $\begin{array}{l}\text { No GDM } \\
n=3,590\end{array}$ & $p$ value $^{\mathrm{a}}$ \\
\hline Age, years & $28(1.4)$ & $28(1.4)$ & 0.10 \\
\hline Area of residence & & & 0.40 \\
\hline Urban & 74.4 & 72.0 & \\
\hline Rural/remote & 25.6 & 28.0 & \\
\hline Highest qualification completed & & & 0.04 \\
\hline Up to year 12 or equivalent & 24.1 & 19.6 & \\
\hline Trade/apprenticeship/certificate/diploma & 26.1 & 22.7 & \\
\hline University/higher degree & 49.8 & 57.7 & \\
\hline Total energy intake, $\mathrm{kJ} /$ day & $6,617.5(2,219.4)$ & $6,591.3(2,195.8)$ & 0.85 \\
\hline Nulliparous & 81.4 & 75.8 & 0.04 \\
\hline Polycystic ovary syndrome & 15.8 & 6.7 & $<0.0001$ \\
\hline BMI, $\mathrm{kg} / \mathrm{m}^{2}$ & $25.8(5.8)$ & $23.7(4.5)$ & $<0.0001$ \\
\hline BMI & & & $<0.0001$ \\
\hline Healthy weight, $<25 \mathrm{~kg} / \mathrm{m}^{2}$ & 54.7 & 71.3 & \\
\hline Overweight, 25 to $<30 \mathrm{~kg} / \mathrm{m}^{2}$ & 25.0 & 19.0 & \\
\hline Obese, $\geq 30 \mathrm{~kg} / \mathrm{m}^{2}$ & 20.3 & 9.7 & \\
\hline Physical activity & & & 0.87 \\
\hline Sedentary/low, $<600 \mathrm{MET}$ min/week & 42.5 & 41.9 & \\
\hline Moderate, 600 to $<1200 \mathrm{MET} \mathrm{min} /$ week & 25.8 & 24.9 & \\
\hline High, $\geq 1200 \mathrm{MET} \mathrm{min} /$ week & 31.7 & 33.2 & \\
\hline Smoking & & & 0.51 \\
\hline Never smoked & 58.6 & 62.1 & \\
\hline Ex-smoker & 19.1 & 17.8 & \\
\hline Current smoker & 22.4 & 20.1 & \\
\hline
\end{tabular}

Values are mean (SD) or \%, weighted by area of residence

${ }^{\text {a }} p$ values from $\chi^{2}$ or $t$ tests
In stratified analyses, the association between the Meats, snacks and sweets pattern and higher GDM risk remained statistically significant in the fully adjusted model for parous ( $p$ value for interaction $=0.05$ ) and obese women $(p=0.11)$ and for women with lower educational qualifications $(p=0.07)$. For each SD increase in dietary pattern score, higher GDM risk was found in parous women (RR 1.92, 95\% CI 1.32, 2.76) (Fig. 2a), women who were obese (RR 2.08, 95\% CI 1.18, 3.62) (Fig. 2b), and women with lower educational qualifications (RR 2.17, 95\% CI 1.14, 4.17) (Fig. 2c). No significant associations were found across strata for polycystic ovary syndrome, smoking and physical activity (all $p$ values for interaction $>0.20$ ).

In the highest tertile of the Meats, snacks and sweets pattern women reported high daily consumption of meat (3 servings), sweet or savoury snacks ( 9 servings) and dairy (mainly high-fat dairy; 2 servings), but also had moderate consumption of vegetables ( 2.5 servings), fruit (1 serving), pasta/rice (2 servings), high-fibre grain foods (such as wholemeal bread, rye bread, muesli; 1 serving) and fish (weekly 2 servings) (ESM Table 2).
Individual food items that highly correlated with the Meats, snacks and sweets pattern and that were associated with risk of developing GDM included fried fish and bacon. Higher consumption of $5 \mathrm{~g} /$ day of fried fish (RR $1.05,95 \% \mathrm{CI} 0.99,1.11$ ) and bacon (RR 1.27, 95\% CI 1.11, 1.46) were associated with higher GDM risk. Eliminating these food items from the dietary pattern slightly attenuated the estimates, but associations remained statistically significant.

Mediterranean-style dietary pattern The Mediterraneanstyle pattern was associated with a $15 \%$ lower risk of developing GDM for each SD increase in score after adjustment for socioeconomic, reproductive and lifestyle factors and BMI (95\% CI 0.76, 0.98) (Table 3). Moreover, a significant linear trend was found across tertiles of dietary pattern scores. Women in the highest tertile had a $44 \%$ lower risk (95\% CI 0.41, 0.77, $p$ for trend 0.0001) when compared with women in the lowest tertile. This association was consistent when stratified by parity, highest qualification completed, polycystic ovary syndrome, smoking, physical activity and BMI (all $p$ values for interaction $>0.20$ ). 


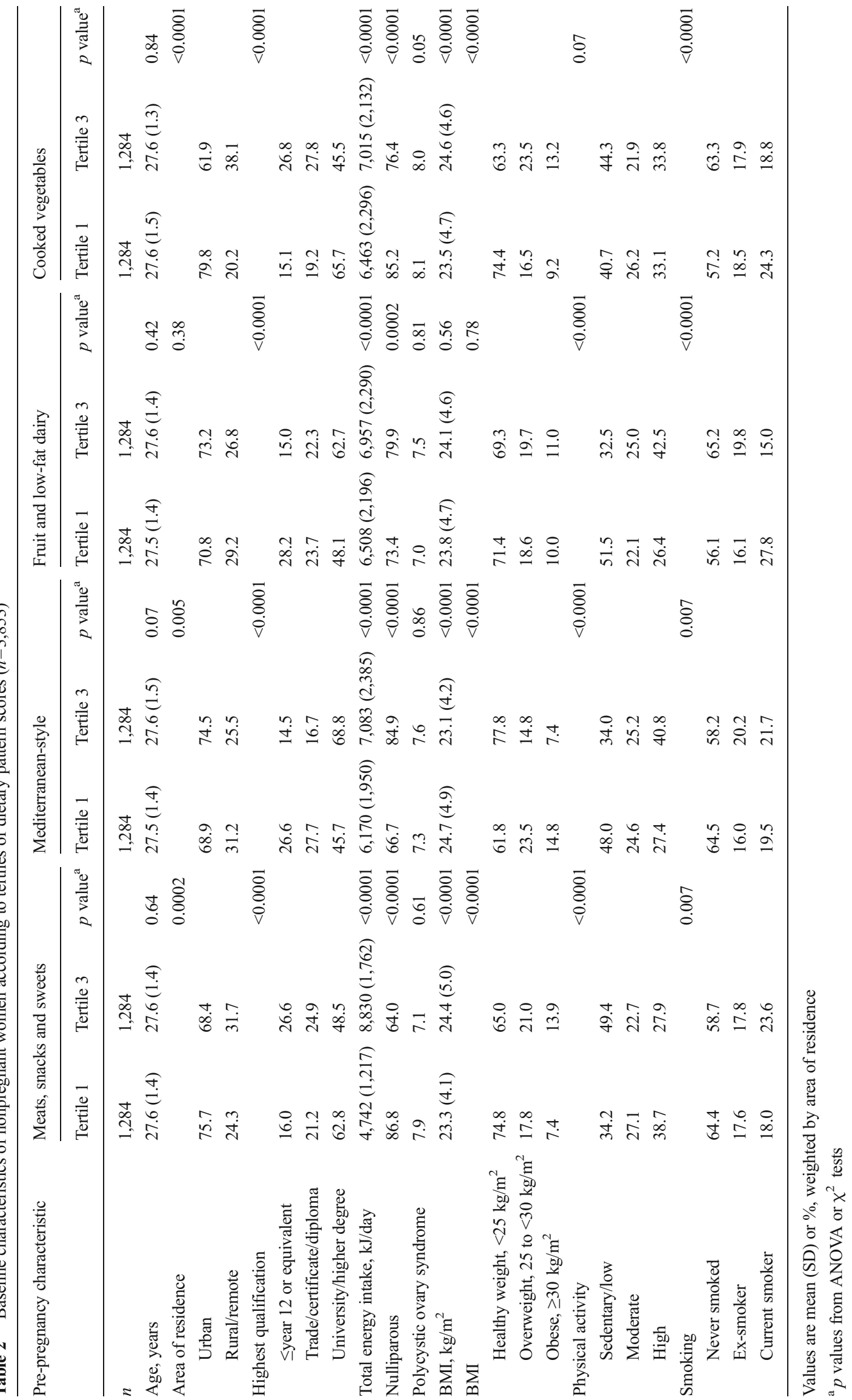


Table 3 Relative risks (95\% CIs) for associations between pre-pregnancy dietary patterns and incidence of GDM $(n=3,853)$

\begin{tabular}{|c|c|c|c|c|c|c|}
\hline \multirow[t]{2}{*}{ Pre-pregnancy dietary pattern } & \multicolumn{4}{|c|}{ Tertiles of dietary pattern scores } & \multirow{2}{*}{$\begin{array}{l}p \text { value for linear } \\
\text { trend }^{\mathrm{a}}\end{array}$} & \multirow{2}{*}{$\begin{array}{l}\text { Continuous (each } 1 \mathrm{SL} \\
\text { increase in score) }\end{array}$} \\
\hline & Tertile 1 & Tertile 2 & Tertile 3 & $p$ value & & \\
\hline \multicolumn{7}{|l|}{ Meats, snacks and sweets } \\
\hline$n$ women/pregnancies & $1,284 / 2,228$ & $1,285 / 2,261$ & $1,284 / 2,137$ & & & $3,853 / 6,626$ \\
\hline GDM cases $n$ ( $\%$ pregnancies $)$ & $89(4.0)$ & $93(4.1)$ & $110(5.1)$ & & & $292(4.4)$ \\
\hline Model $1^{\mathrm{b}}$ & 1.00 & $1.06(0.76,1.49)$ & $1.30(0.81,2.08)$ & 0.51 & 0.28 & $1.41(1.03,1.91)$ \\
\hline Model $2^{\mathrm{c}}$ & 1.00 & $1.07(0.76,1.51)$ & $1.28(0.80,2.05)$ & 0.56 & 0.30 & $1.38(1.02,1.86)$ \\
\hline Model $3^{\mathrm{d}}$ & 1.00 & $1.03(0.74,1.45)$ & $1.23(0.76,1.97)$ & 0.65 & 0.39 & $1.35(0.98,1.81)$ \\
\hline \multicolumn{7}{|l|}{ Mediterranean-style } \\
\hline$n$ women/pregnancies & $1,284 / 2,127$ & $1,285 / 2,250$ & $1,284 / 2,249$ & & & $3,853 / 6,626$ \\
\hline GDM cases $n$ ( $\%$ pregnancies $)$ & $119(5.6)$ & $108(4.8)$ & $65(2.9)$ & & & $292(4.4)$ \\
\hline Model $1^{\mathrm{b}}$ & 1.00 & $0.94(0.72,1.24)$ & $0.54(0.39,0.74)$ & 0.0001 & $<0.0001$ & $0.86(0.74,0.99)$ \\
\hline Model $2^{\mathrm{c}}$ & 1.00 & $0.93(0.71,1.20)$ & $0.52(0.38,0.71)$ & $<0.0001$ & $<0.0001$ & $0.86(0.73,0.98)$ \\
\hline Model $3^{\mathrm{d}}$ & 1.00 & $0.97(0.75,1.28)$ & $0.56(0.41,0.77)$ & 0.0003 & 0.0001 & $0.85(0.76,0.98)$ \\
\hline \multicolumn{7}{|l|}{ Fruit and low-fat dairy } \\
\hline$n$ women/pregnancies & $1,284 / 2,121$ & $1,285 / 2,222$ & $1,284 / 2,283$ & & & \\
\hline GDM cases $n$ ( $\%$ pregnancies $)$ & $98(4.6)$ & $101(4.5)$ & $93(4.1)$ & & & \\
\hline Model $1^{\mathrm{b}}$ & 1.00 & $1.13(0.84,1.51)$ & $1.03(0.76,1.39)$ & 0.69 & & \\
\hline Model $2^{\mathrm{c}}$ & 1.00 & $1.15(0.85,1.54)$ & $1.04(0.77,1.42)$ & 0.67 & & \\
\hline Model $3^{\mathrm{d}}$ & 1.00 & $1.12(0.83,1.49)$ & $1.01(0.76,1.37)$ & 0.75 & & \\
\hline \multicolumn{7}{|l|}{ Cooked vegetables } \\
\hline$n$ women/pregnancies & $1,284 / 2,254$ & $1,285 / 2,247$ & $1,284 / 2,125$ & & & \\
\hline GDM cases $n$ ( $\%$ pregnancies $)$ & $105(4.7)$ & $82(3.6)$ & $105(4.9)$ & & & \\
\hline Model $1^{\mathrm{b}}$ & 1.00 & $0.83(0.61,1.13)$ & $1.06(0.80,1.41)$ & 0.27 & & \\
\hline Model $2^{\mathrm{c}}$ & 1.00 & $0.84(0.62,1.15)$ & $1.08(0.81,1.43)$ & 0.27 & & \\
\hline Model $3^{\mathrm{d}}$ & 1.00 & $0.83(0.61,1.13)$ & $1.04(0.77,1.38)$ & 0.38 & & \\
\hline
\end{tabular}

${ }^{a}$ If a linear trend was evident from estimates across tertiles of dietary pattern scores, a $p$ value for linear trend was determined and dietary patterns were in addition analysed as continuous scores

${ }^{\mathrm{b}}$ Adjusted for age (years), total energy intake (kJ/day), highest qualification completed (up to year 12 or equivalent, trade/apprenticeship/certificate/ diploma, university/higher degree), parity (nulliparous [no previous births], parous [given birth at least once]), hypertensive disorders of pregnancy (yes, no), polycystic ovary syndrome (yes, no), inter-pregnancy interval (not applicable [first pregnancy], $<18$ months, 18-60 months, $>60$ months)

${ }^{\mathrm{c}}$ Additionally adjusted for smoking (never smoked, exsmoker, current smoker), physical activity (sedentary/low, moderate, high)

${ }^{\mathrm{d}}$ Additionally adjusted for BMI (healthy weight, overweight, obese)

Women in the highest tertile of the Mediterranean-style dietary pattern daily consumed on average 2.5 servings of vegetables, 1.5 servings of fruits, 2 servings of dairy (mainly low-fat), 2 servings of pasta/rice, and weekly 2 servings of fish (ESM Table 2). Compared with women in the highest tertile of the Meats, snacks and sweets dietary pattern, women in the highest tertile of the Mediterranean-style dietary pattern had higher daily consumption of high-fibre grain foods ( 2 servings), and lower consumption of meat (1.5 servings) and sweet or savoury snacks (4 servings).

Consumption of capsicum (bell or sweet peppers) (RR $0.75,95 \%$ CI $0.58,0.98$ ), red wine (RR $0.98,95 \%$ CI 0.97 , 0.99 ) and rye bread (RR $0.96,95 \%$ CI $0.90,0.98$ ) were highly correlated with the Mediterranean-style pattern and associated with lower risk of GDM for each increase of $5 \mathrm{~g} /$ day. Excluding these food items from the Mediterraneanstyle dietary pattern attenuated the estimates slightly, but did not remove the statistically significant association with GDM.

Associations between the Meats, snacks and sweets and the Mediterranean-style dietary patterns and GDM persisted in sensitivity analyses: similar results were observed for the associations between dietary patterns based on average longterm pre-pregnancy diet and GDM risk, and in analysis in which missing covariate data were imputed (data not shown).

\section{Discussion}

In this population-based prospective cohort study of Australian women, a pre-pregnancy Meats, snacks and sweets 

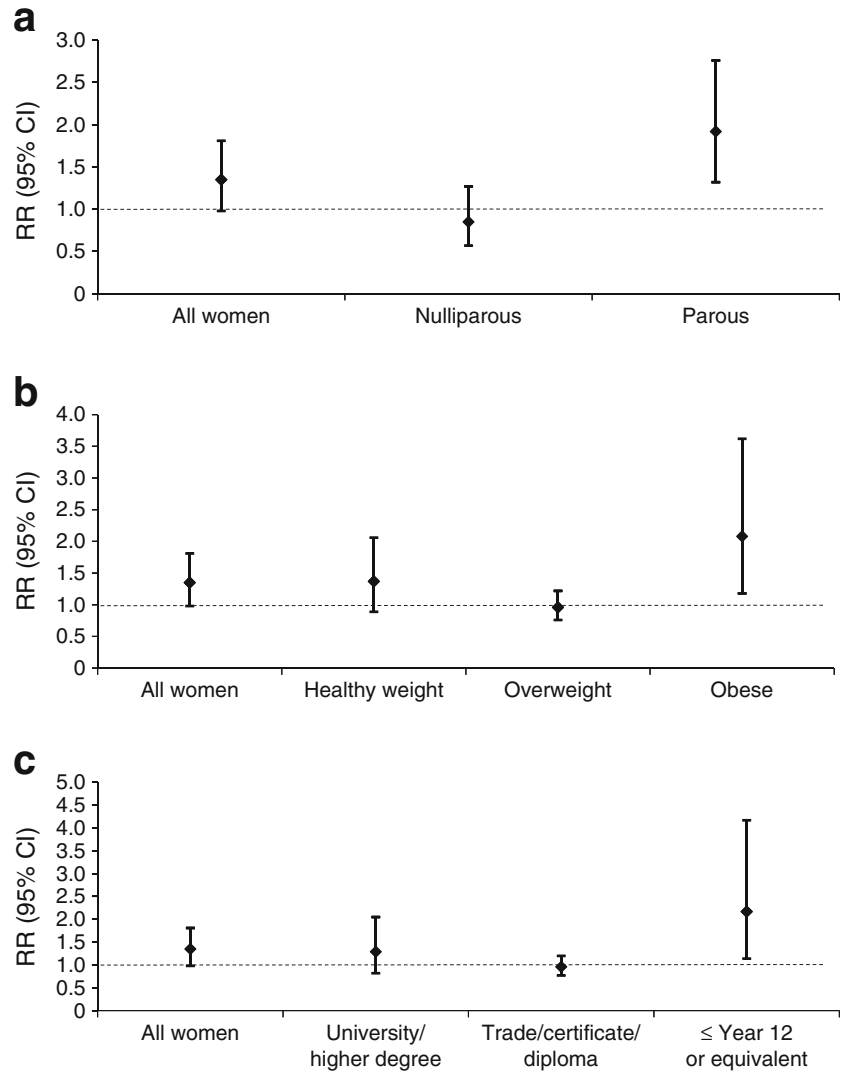

Fig. 2 Association between the pre-pregnancy Meats, snacks and sweets dietary pattern (per 1 SD increase in score) and incidence of GDM stratified by (a) parity ( $p$ value for interaction $=0.05),(\mathbf{b})$ BMI $(p=0.11)$ and (c) highest qualification completed $(p=0.07)$. Estimates are adjusted for age, total energy intake, highest qualification completed, parity, hypertensive disorders of pregnancy, polycystic ovary syndrome, inter-pregnancy interval, smoking, physical activity and BMI

dietary pattern, characterised by high consumption of red and processed meat and snacks, was associated with higher GDM risk after controlling for socioeconomic, reproductive and lifestyle factors. After additional adjustment for BMI, this association remained statistically significant only for parous and obese women, and women with lower educational qualifications. Moreover, we observed an inverse dose-response association between a pre-pregnancy Mediterranean-style dietary pattern, characterised by high consumption of vegetables, whole grains, nuts and fish, and lower risk of developing GDM independent of other risk factors. No associations were found for the Fruit and low-fat dairy and the Cooked vegetables dietary patterns.

Evidence from previous studies on pre-pregnancy dietary patterns and GDM is limited to findings from the Nurses' Health Study II [13-15]. High adherence to a 'Western' dietary pattern, and low adherence to a 'Prudent' dietary pattern and a priori defined healthful dietary pattern scores (alternate Mediterranean Diet [aMED], Dietary Approaches to Stop Hypertension [DASH] and alternate Healthy Eating Index [aHEI]), were associated with higher GDM risk [14, 15]. The 'Prudent' dietary pattern and the healthful dietary pattern scores were all characterised by high intake of fruit, vegetables, legumes, nuts, whole grains and fish, and low intake of red and processed meat. Even though the combination of foods and the specific labelling of the patterns were different across our studies, findings in this population of US nurses were generally consistent with our results in a random community sample of reproductive-aged Australian women. By contrast, however, attenuation of effect estimates after confounding adjustment appeared to be driven by BMI, but associations observed in the Nurses' Health Study II remained statistically significant in final models $[14,15]$. In our study, the Mediterranean-style dietary pattern was significantly associated with lower GDM risk after controlling for confounders including BMI; however, the association between the Meats, snacks and sweets dietary pattern and higher GDM risk was explained by BMI in the overall study population. Higher consumption of foods that are high in energy, saturated fat and sugar is a risk factor for obesity, which in turn is a strong risk factor for GDM. BMI might, therefore, be an intermediate in the association between the Meats, snacks and sweets pattern and GDM risk.

In stratified analysis, the association between the Meats, snacks and sweets pattern and higher risk of GDM remained significant after adjustment for other risk factors for subgroups of women, including parous and obese women, and women with low educational qualifications. In line with most previous studies [32-34], these subgroups of women were found to have a less healthy diet compared with nulliparous women, women with a healthy weight and with higher educational qualifications. The higher consumption of red and processed meat, sweets and snacks by these women might explain the association with a significantly higher risk of GDM. In addition, a range of other factors such as less social support, higher stress levels and less healthy food preparation methods may have altered the association between pre-pregnancy dietary patterns and GDM in these high-risk groups.

Mechanisms underlying the associations between the Meats, snacks and sweets and the Mediterranean-style dietary patterns and GDM risk are not clear and remain speculative, but may include influencing oxidative stress and inflammatory markers as potential pathways involved in the pathogenesis of GDM [35]. Foods high in sugar and saturated fat have been shown to promote oxidative stress and inflammation [36, 37]. By contrast, vegetables, legumes, nuts and whole grains are low in energy and fat and have a high content of dietary fibre, magnesium, vitamin $\mathrm{E}$ and other antioxidants that may contribute to reducing markers of oxidative stress and inflammation [36-38]. It remains unclear whether these potential pathways linking diet with GDM are independent of BMI, as oxidative stress and inflammation have been suggested as processes that may link adiposity with the development of GDM [39]. Further study is needed to elucidate the effects and inter-relationships between diet, oxidative stress, inflammation, adiposity and development of GDM. 
A unique feature of our study is that data were collected on women before pregnancy, whether or not they were planning a pregnancy. The longitudinal design enables the examination of prospective associations with risk of developing GDM. Results from this nationally representative population-based sample are generalisable to the Australian population of reproductive-aged women [16]. Moreover, examining dietary patterns rather than individual nutrients and foods enabled the capture of potential joined effects of all components of a diet. We found that no individual food could fully explain the observed associations, suggesting that the combination and interaction of nutrients and foods of the Meats, snacks and sweets and the Mediterranean-style dietary patterns were essential for the observed associations with GDM risk.

Some limitations of our study should be acknowledged. First, misclassification is a concern in self-reported dietary assessment. Owing to the prospective design of our study, however, reporting of dietary intake could not have been biased by the subsequent development of GDM. The FFQ used in this study has been validated and shown to be a valuable tool for assessing habitual dietary intake [20]. Food availability and consumption patterns may have changed since the collection of dietary data in our study in 2003 and 2009. Consumption of certain foods not included in our FFQ, such as lean meats, unsweetened low-fat dairy, a wide range of soy foods, legumes, nuts and seeds, and low-carbohydrate, highprotein snacks, should be examined in future studies. Second, dietary intake specifically during pregnancy was not measured in our study. However, evidence from prospective cohort studies suggests that dietary patterns do not change significantly before, during and after pregnancy [40, 41]. Further studies are needed to examine the independent and combined effects of diet before and during pregnancy with risk of GDM. Third, misclassification of GDM cases and non-cases may have introduced bias; however, reliability of self-reported GDM diagnosis in our study population showed high agreement with administrative records. Australia has a standard of universal GDM screening of all women [24], minimising the risk of missing cases. Last, causal relationships cannot be inferred from our observational data. Associations were adjusted for a variety of known risk factors of GDM, but we were not able to control for factors such as family history of diabetes or GDM, and gestational and inter-pregnancy weight gain. These unmeasured factors may have introduced residual confounding.

In conclusion, our results indicate that higher prepregnancy consumption of foods in the Mediterranean-style dietary pattern is associated with lower risk of developing GDM. Moreover, higher pre-pregnancy consumption of foods in the Meats, snacks and sweets dietary pattern is associated with higher GDM risk, particularly in high-risk groups including parous and obese women, and women with lower educational qualifications. These findings support general dietary recommendations for women of reproductive age to consume a diet rich in vegetables, legumes, whole grains, nuts and fish, and low in red and processed meat, snacks and sweets $[42,43]$. Further large prospective observational and intervention studies in a range of populations are needed to confirm whether dietary patterns both before and during pregnancy reduce the risk of GDM.

Acknowledgements The authors thank G. Giles of the Cancer Epidemiology Centre of The Cancer Council Victoria for permission to use the Dietary Questionnaire for Epidemiological Studies, version 2 (The Cancer Council Victoria, Melbourne, 1996). We also thank all the participants for their valuable contribution to this project.

Funding The Australian Longitudinal Study on Women's Health was conceived and developed at the Universities of Newcastle and Queensland and is funded by the Australian Government Department of Health. DAJMS is supported by an International Postgraduate Research Scholarship and GDM by an Australian Research Council Future Fellowship (FT120100812).

Duality of interest The authors declare that there is no duality of interest associated with their contribution to this manuscript.

Contribution statement DAJMS designed the research, performed the statistical analysis, wrote the paper and had primary responsibility for the final content. SSS-M and GDM contributed to design of the research, interpretation of the results and critical revision of the manuscript for important intellectual content. LKC contributed to interpretation of the results and critical revision of the manuscript for important intellectual content. All authors approved the final version to be published. DAJMS is the guarantor of this work.

\section{References}

1. American Diabetes Association (2013) Diagnosis and classification of diabetes mellitus. Diabetes Care 36:S67-S74

2. Australian Institute of Health and Welfare (2010) Diabetes in pregnancy: its impact on Australian women and their babies. In: Diabetes series no. 14. Cat. no. CVD 52. Australian Institute of Health and Welfare, Canberra

3. Dabelea D, Snell-Bergeon JK, Hartsfield CL, Bischoff KJ, Hamman RF, McDuffie RS (2005) Increasing prevalence of gestational diabetes mellitus (GDM) over time and by birth cohort: Kaiser Permanente of Colorado GDM Screening Program. Diabetes Care 28:579-584

4. Guberman C, Kjos SL (2010) Maternal comorbidities during gestational diabetes mellitus: obstetrical complications, prematurity, and delivery. In: Kim C, Ferrara A (eds) Gestational diabetes during and after pregnancy. Springer-Verlag, London, pp 215-226

5. Kim C, Newton KM, Knopp RH (2002) Gestational diabetes and the incidence of type 2 diabetes: a systematic review. Diabetes Care 25:1862-1868

6. Dabelea D (2010) The diabetic intrauterine environment: short and long-term consequences. In: Kim C, Ferrara A (eds) Gestational diabetes during and after pregnancy. Springer-Verlag, London, pp 227-239

7. Egeland GM, Skjærven R, Irgens LM (2000) Birth characteristics of women who develop gestational diabetes: population based study. BMJ 321:546-547

8. Facchinetti F, Dante G, Petrella E, Neri I (2014) Dietary interventions, lifestyle changes, and dietary supplements in preventing 
gestational diabetes mellitus: a literature review. Obstet Gynecol Surv 69:669-680

9. Rogozińska E, Chamillard M, Hitman GA, Khan KS, Thangaratinam S (2015) Nutritional manipulation for the primary prevention of gestational diabetes mellitus: a meta-analysis of randomised studies. PLoS One 10:e0115526

10. Schoenaker DA, Mishra GD, Callaway LK, Soedamah-Muthu SS (2015) The role of energy, nutrients, foods and dietary patterns in the development of gestational diabetes mellitus: a systematic review of observational studies. Diabetes Care. doi:10.2337/DC150540

11. De-Regil LM, Fernández-Gaxiola AC, Dowswell T, Peña-Rosas JP (2010) Effects and safety of periconceptional folate supplementation for preventing birth defects. Cochrane Database Syst Rev, issue 10, Art. no.: CD007950. doi:10.1002/14651858.CD007950.pub2

12. Torloni M, Betrán A, Horta B et al (2009) Prepregnancy BMI and the risk of gestational diabetes: a systematic review of the literature with meta-analysis. Obes Rev 10:194-203

13. Bao W, Bowers K, Tobias DK et al (2014) Prepregnancy lowcarbohydrate dietary pattern and risk of gestational diabetes mellitus: a prospective cohort study. Am J Clin Nutr 99:1378-1384

14. Tobias DK, Zhang C, Chavarro J et al (2012) Prepregnancy adherence to dietary patterns and lower risk of gestational diabetes mellitus. Am J Clin Nutr 96:289-295

15. Zhang C, Schulze MB, Solomon CG, Hu FB (2006) A prospective study of dietary patterns, meat intake and the risk of gestational diabetes mellitus. Diabetologia 49:2604-2613

16. Brown WJ, Bryson L, Byles JE et al (1998) Women's Health Australia: recruitment for a national longitudinal cohort study. Women Health 28:23-40

17. Lee C, Dobson AJ, Brown WJ et al (2005) Cohort profile: the Australian longitudinal study on women's health. Int J Epidemiol 34:987-991

18. Willett WC (1998) Nutritional epidemiology. Oxford University Press, New York

19. Ireland P, Jolley D, Giles G et al (1994) Development of the Melbourne FFQ: a food frequency questionnaire for use in an Australian prospective study involving an ethnically diverse cohort. Asia Pac J Clin Nutr 3:19-31

20. Hodge A, Patterson AJ, Brown WJ, Ireland P, Giles G (2000) The Anti Cancer Council of Victoria FFQ: relative validity of nutrient intakes compared with weighed food records in young to middle-aged women in a study of iron supplementation. Aust N Z J Public Health 24:576583

21. Ocke MC, Bueno-de-Mesquita HB, Pols MA, Smit HA, van Staveren WA, Kromhout D (1997) The Dutch EPIC food frequency questionnaire. II. Relative validity and reproducibility for nutrients. Int J Epidemiol 26:S49-S58

22. Patterson RE, Kristal AR, Tinker LF, Carter RA, Bolton MP, AgursCollins T (1999) Measurement characteristics of the Women's Health Initiative food frequency questionnaire. Ann Epidemiol 9: 178-187

23. Lewis J, Milligan GC, Hunt A (1995) Nuttab95: Nutrient data table for use in Australia. Australian Government Publishing Service, Canberra

24. Hoffman L, Nolan C, Wilson JD, Oats J, Simmons D (1998) Gestational diabetes mellitus-management guidelines. The Australasian Diabetes in Pregnancy Society. Med J Aust 169:93-97

25. Gresham E, Forder P, Chojenta CL, Byles JE, Loxton DE, Hure AJ (2015) Agreement between self-reported perinatal outcomes and administrative data in New South Wales, Australia. BMC Pregnancy Childbirth 15:161-170

26. Brown WJ, Burton NW, Marshall AL, Miller YD (2008) Reliability and validity of a modified self-administered version of the Active Australia physical activity survey in a sample of mid-age women. Aust N Z J Public Health 32:535-541

27. McCann SE, Marshall JR, Brasure JR, Graham S, Freudenheim JL (2001) Analysis of patterns of food intake in nutritional epidemiology: food classification in principal components analysis and the subsequent impact on estimates for endometrial cancer. Public Health Nutr 4:989-997

28. Kline P (1994) An easy guide to factor analysis. Routledge, London

29. Hanley JA, Negassa A, Forrester JE (2003) Statistical analysis of correlated data using generalized estimating equations: an orientation. Am J Epidemiol 157:364-375

30. Hu FB, Stampfer MJ, Rimm E et al (1999) Dietary fat and coronary heart disease: a comparison of approaches for adjusting for total energy intake and modeling repeated dietary measurements. Am J Epidemiol 149:531-540

31. Berglund PA (2010) An introduction to multiple imputation of complex sample data using SAS v9.2. In: SAS Global Forum Proceedings. SAS Institute Inc, Cary, NC

32. Elstgeest LE, Mishra GD, Dobson AJ (2012) Transitions in living arrangements are associated with changes in dietary patterns in young women. J Nutr 142:1561-1567

33. Galobardes B, Morabia A, Bernstein MS (2001) Diet and socioeconomic position: does the use of different indicators matter? Int J Epidemiol 30:334-340

34. Togo P, Osler M, Sørensen T, Heitmann B (2001) Food intake patterns and body mass index in observational studies. Int J Obes Relat Metab Disord 25:1741-1751

35. McCurdy CE, Friedman JE (2010) Mechanisms underlying insulin resistance in human pregnancy and gestational diabetes mellitus. In: Kim C, Ferrara A (eds) Gestational diabetes during and after pregnancy. Springer-Verlag, London, pp 125-138

36. Fung TT, McCullough ML, Newby P et al (2005) Diet-quality scores and plasma concentrations of markers of inflammation and endothelial dysfunction. Am J Clin Nutr 82:163-173

37. Lopez-Garcia E, Schulze MB, Fung TT et al (2004) Major dietary patterns are related to plasma concentrations of markers of inflammation and endothelial dysfunction. Am J Clin Nutr 80:1029-1035

38. Dai J, Jones DP, Goldberg J et al (2008) Association between adherence to the Mediterranean diet and oxidative stress. Am J Clin Nutr 88:1364-1370

39. Retnakaran R (2010) Inflammation, adipokines, and gestational diabetes mellitus. In: Kim C, Ferrara A (eds) Gestational diabetes during and after pregnancy. Springer-Verlag, London, pp 139-153

40. Cuco G, Fernandez-Ballart J, Sala J et al (2005) Dietary patterns and associated lifestyles in preconception, pregnancy and postpartum. Eur J Clin Nutr 60:364-371

41. McGowan CA, McAuliffe FM (2013) Maternal dietary patterns and associated nutrient intakes during each trimester of pregnancy. Public Health Nutr 16:97-107

42. National Health and Medical Research Council (2013) Australian dietary guidelines. National Health and Medical Research Council, Canberra

43. US Department of Agriculture and US Department of Health and Human Services (2010) Dietary Guidelines for Americans, 2010. Government Printing Office, Washington, DC 